\title{
Cirugía hipofisiaria endoscópica transesfenoidal, con realización de colgajo nasoseptal: Evaluación del impacto de la técnica en la olfación. Serie de casos
}

\author{
Endoscopic pitituary surgery transsphenoidal, with nasoseptal flap: \\ Assessment of the impact of the technique in the olfaction. Cases series
}

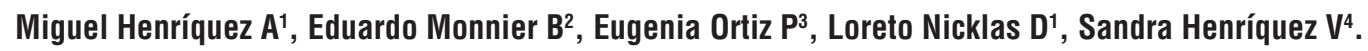

\begin{abstract}
RESUMEN
Introducción: La cirugía endoscópica transesfenoidal en la exéresis de patología hipofisaria ha sido ratificada dentro de la mejores opciones para conseguir abordajes exitosos. Para cerrar la brecha ósea, se puede utilizar el colgajo nasoseptal, el cual lograría un cierre adecuado y seguro, evitando complicaciones como persistencia de fístulas de líquido cefalorraquídeo. Dentro de los probables efectos secundarios de este colgajo se señala la presencia de anosmia o hiposmia.

Objetivo: Nuestro objetivo es mostrar los resultados obtenidos empleando la técnica del colgajo nasoseptal y la evaluación del olfato posoperatorio.

Material y método: Incluimos 14 pacientes con diagnóstico de adenoma hipofisiario intervenidos en nuestro servicio entre diciembre 2014 a diciembre 2015.

Se evaluaron diversos parámetros entre otros, olfatometría pre y posoperatorias.

A todos se les realizó la técnica endoscópica transesfenoidal. Efectuando la disección del colgajo con la técnica tipo Hadad. La exéresis tumoral fue realizada por neurocirujano, se cubrió la osteotomía con este colgajo.

Resultados: De los 14 pacientes, 13 llegaron al estado olfativo preoperatorio. 1 paciente se mantuvo hipósmico mantenidamente.

En nuestro estudio, no hemos encontrado deterioro significativo en la calidad del sentido del olfato. Así como tampoco observamos otras complicaciones.

Conclusiones: Esta experiencia aún inicial, estimamos que podría ya esbozar una tendencia de mantención de la función olfativa en este tipo de cirugía.

Palabras clave: Colgajo nasoseptal pediculado, cirugía endonasal de base de cráneo, endoscópico, función olfatoria.
\end{abstract}

\footnotetext{
Médico Otorrinolaringólogo. Departamento de Especialidades Médicas. Universidad de la Frontera. Temuco.

Médico Neurocirujano. Servicio de Neurocirugía. Hospital Hernán Henríquez Aravena. Temuco.

Médico Endocrinólogo. Departamento de Medicina Interna. Universidad de la Frontera. Temuco.

4 Tecnólogo Médico Mención ORL. Servicio de Otorrinolaringología. Hospital Hernán Henríquez Aravena. Temuco.
}

Recibido el 6 de septiembre, 2016. Aceptado el 20 de octubre, 2016. 


\section{ABSTRACT}

Introduction: Transsphenoidal endoscopic surgery resection of pituitary pathology has been ratified within the best options for successful approaches.

To close the gap bone, you can use the nasoseptal flap, which achieved an adequate and secure closure, preventing complications such as persistent cerebrospinal fluid leaks. Among possible side effects of this flap anosmia-hyposmia noted.

Aim: Our goal is to show the results obtained using the technique of nasoseptal flap and postoperative evaluation of smell.

Material and method: We included 14 patients with a diagnosis of pituitary adenoma surgery in our department between December 2014 to December 2015.

Various parameters were evaluated pre and post operative, specially olfactory function. All underwent transsphenoidal endoscopic technique. Performing dissection flap with the technical type Hadad. The tumor resection was performed by neurosurgeon, osteotomy covered with this flap.

Results: Of the 14 patients, 13 reached the olfactory preoperative state. 1 patient remained with hyposmia. In our study, we found no significant deterioration in the quality of the sense of smell. Not other complications were found.

Conclusions: This is an initial experience, and we estimate that could outline a trend of maintaining olfactory function in this surgery approach.

Key words: Nasoseptal flap, endonasal endoscopic surgery of skull base, endoscopy, olfactory function.

\section{INTRODUCCIÓN}

La cirugía endoscópica transesfenoidal en la exéresis de patología hipofisaria ha sido ratificada como una de la mejores opciones para conseguir un abordaje exitoso, su aplicación en esta patología fue iniciada en 1995 por Sethi', Jho $^{2}$ y Carrau ${ }^{3}$, entre otros.

A través de esta técnica, se logra optimizar la visualización de las estructuras, asegurando una mejor resección tumoral. Así, gracias a la colaboración mutua entre otorrinolaringólogos y neurocirujanos y aprovechando la experiencia de este abordaje por los primeros, se han obtenido buenas experiencias en numerosos equipos ${ }^{4,5}$.

A nivel nacional, existen sendas experiencias publicadas por diferentes grupos, tanto microscópicas como endoscópicas ${ }^{6-8}$.

Para el cierre de la brecha ósea, entre otras alternativas, se ha empleado la utilización de un colgajo nasoseptal, el cual lograría un cierre adecuado y seguro del defecto originado. Logrando evitar una de las complicaciones más importantes como es la persistencia de fístulas de líquido cefalorraquídeo.
La realización del colgajo nasoseptal fue descrito inicialmente por $\operatorname{Hadad}^{9}$, con algunas modificaciones posteriores ${ }^{10}$. Es un colgajo de mucoperiostio vascularizado por ramas de las arterias nasoseptales, proveniente de la arteria nasal posterior, estas arterias irrigan todo el septo nasal anastomosándose con las arterias etmoidales anteriores en la parte superior, la arteria palatina mayor en la parte inferior y la arteria facial en la parte anterior. Este pedículo ricamente vascularizado le otorga a este colgajo una gran versatilidad, viabilidad, y un arco de rotación con una gran área potencial de recubrimiento.

Durante la cirugía hipofisiaria, efectuar este colgajo, no representa una gran dificultad técnica y no alarga excesivamente la intervención.

Dentro de las dificultades técnicas al llevar a cabo la resección de tumores hipofisiarios bajo la técnica endoscópica y en los casos que se efectúe el colgajo nasoseptal, podemos señalar que la pérdida del olfato es una de los efectos secundarios recurrentemente mencionados. Esto se debe principalmente a que las áreas mucoseptales involucradas en la propia intervención o en la manipulación de éstas, podrían llevar a una afectación 
de las funciones olfativas ${ }^{11,12}$. Durante la realización de la esfenoidotomía o el actuar sobre el septum posterior, este colgajo podría resultar lesionado. Así mismo, el instrumental con que se realiza este colgajo, ejemplo bisturí eléctrico aumentaría esta hiposmia ${ }^{13}$. Todo esto, conlleva posteriormente a un detrimento en la calidad de vida para el paciente ${ }^{14}$.

\section{OBJETIVO}

El objetivo de este estudio es mostrar los resultados obtenidos empleando la técnica del colgajo nasoseptal y su resultado en la evaluación del olfato posoperatorio.

\section{MATERIAL Y MÉTODO}

El diseño de este estudio fue un estudio descriptivo de una serie de casos.

La población estudiada fueron 14 pacientes con diagnóstico de adenoma hipofisiario, cuyo diagnóstico fue confirmado con pruebas de imágenes, TAC y RMN. Se realizó examen endoscópico y clínico. Un comité interdisciplinario compuesto por endocrinólogos, neurocirujanos, neurorradiólogos y otorrinolaringólogos confirmó el diagnóstico.

El estudio fue realizado en la Unidad de Otorrinolaringología y Neurocirugía del Hospital Dr. Hernán Henríquez Aravena, Temuco, Chile; en el período de diciembre de 2014 a diciembre 2015.

Como criterios de inclusión se incluyeron todos los pacientes que fueron evaluados por el comité interdisciplinario y aceptados para ser intervenidos quirúrgicamente.

Como criterios de exclusión se consideró la existencia de la presencia de anosmia previa, secundaria a patologías rinogénicas 0 a cirugía hipofisiaria previa.

El estudio de variables consideró los siguientes factores: edad, sexo, cirugía hipofisiaria previa, tamaño tumoral, realización de colgajo nasoseptal, y en especial evaluación de olfatometría pre y posoperatorias.

El Comité de Ética del Hospital Hernán Henríquez Aravena de Temuco aprobó el estudio. Los pacientes firmaron el consentimiento informado para la intervención y para ser partícipes de este estudio, no existiendo cuestionamiento de ninguno de ellos.

El protocolo de trabajo fue el siguiente: se les aplicó la técnica endoscópica transesfenoidal. Inicialmente y durante el mismo acto operatorio el otorrinolaringólogo realizó eventuales correcciones de patologías nasosinusales, como desviaciones septales, conchas bullosas, hipertrofia cornetes. A continuación se llevó a cabo la disección del colgajo de mucosa septal bajo visión endoscópica. Este colgajo se elaboró disecando el mucoperiostio y mucopericondrio desde el borde libre de la coana hasta el septum nasal, el cual recibe nutrición de la arteria nasoseptal. Se disecó en su límite superior aproximadamente $3 \mathrm{~cm}$ bajo la lámina cribosa y como límite inferior la cresta maxilar o el piso de la fosa, siguiendo la técnica descrita por Hadad ${ }^{9}$ (Figuras de edición del autor 1, 2, 3 y 4).

Al finalizar la exéresis tumoral, la cual fue realizada por el neurocirujano, se cubrió la brecha ósea posicionando el colgajo, sobre cartílago septal en 3 ocasiones y en el resto directamente sobre la superficie ósea, se selló empleando Beriplast $^{\mathrm{B}}$. Se taponaron ambas fosas con Merocell $^{\mathrm{M}}$, Ios cuales fueron retirados entre 5 a 7 días posoperatorio.

Como protocolo de medición se aplicó la técnica denominada olfatómetro práctico, técnica desarrollada por un equipo médico del Hospital Sótero del Río, Santiago, Chile ${ }^{15}$. En esta técnica se miden tres parámetros clínico: umbral olfatorio, identificación de diferentes olores y discriminación de diversas concentraciones de un mismo olor; clasificando los resultados en:

1. Pacientes anósmicos (0 puntos).

2. Pacientes hipósmicos (1 a 5 puntos).

3. Pacientes normósmicos ( 6 a 7 puntos).

El análisis se realizó preoperatoriamente, al primer mes, al tercer mes y los seis meses de posoperatorio.

\section{RESULTADOS}

De los 14 pacientes, 7 eran de sexo masculino y 7 femeninos, con un promedio de edad de 50 años (35-66). En relación al tipo de tumores, 


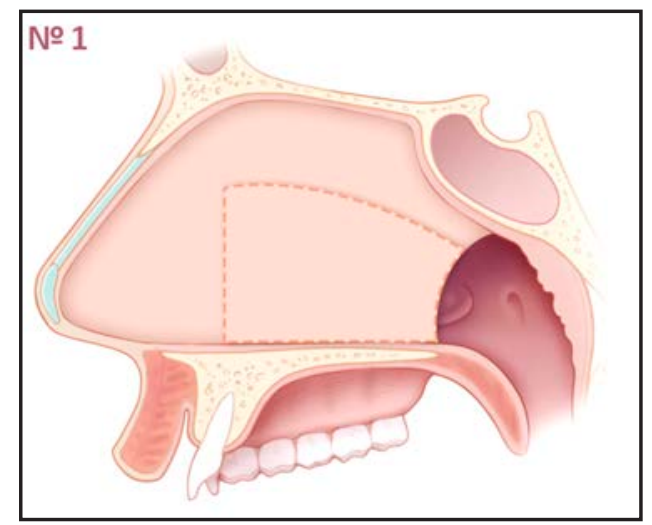

Figura 1. Esquema donde se diseña el futuro colgajo nasoseptal.

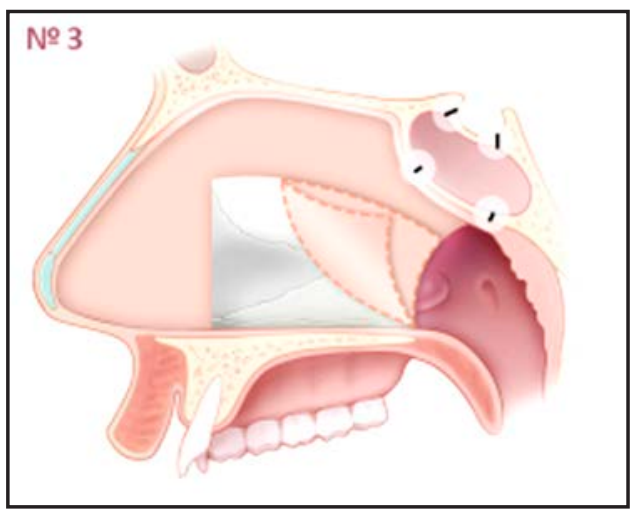

Figura 3. Brecha ósea en pared anterior y posterior de seno esfenoidal.

intervenimos 13 pacientes con macroadenomas y 1 paciente con microadenoma funcionante.

De éstos, 4 pacientes habían sido intervenidos previamente, 3 en una ocasión vía transcraneal, otro en dos oportunidades bajo esta misma vía. En todos estos casos, en sus controles

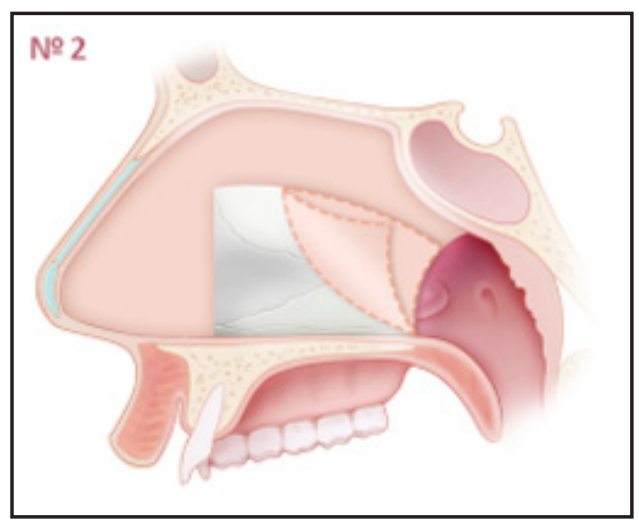

Figura 2. Elaboración de colgajo nasoseptal, elevación mucopericondrio y mucoperiostio.

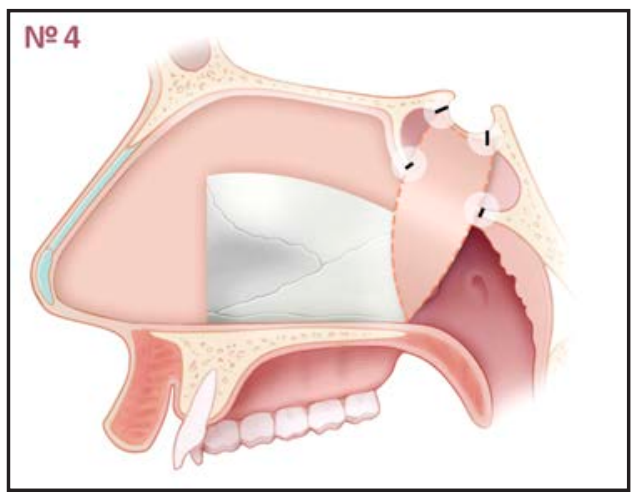

Figura 4. Rotación del colgajo y cierre del defecto óseo.

sucesivos se comprobó la presencia de recidivas tumorales.

Previamente excluimos de esta revisión a 2 pacientes quienes habían sido sometidos a cirugías de resección hipofisiaria previas y presentaron en la evaluación olfatométrica anosmia (índice 0).

Tabla 1. Sistema de puntuación del Olfatómetro Práctico. Tomado de Carolina Der M, y col. Propuesta de una nueva herramienta clínica. Rev Otorrinolaringol Cir Cab-Cuello 2002; 62: 129-36

\begin{tabular}{|cll|}
\hline Umbral olfatorio & Identificación de olores & Discriminación de concentraciones \\
\hline No detecta: 0 punto & respuesta correcta: 1 punto & No discrimina: 0 punto \\
$0-15$ cms: 1 punto & respuesta correcta: 1 punto & Discrimina: 1 punto \\
$>15$ cms: 2 puntos & respuesta correcta: 1 punto & \\
& respuesta correcta: 1 punto & \\
\hline
\end{tabular}


En 2 casos se tuvo que realizar septoplastía al comenzar la intervención. Así como también en 5 ocasiones se llevó a cabo turbinectomía media parcial a fin de optimizar la visualización del ostium esfenoidal.

El colgajo nasoseptal se llevó a cabo de acuerdo a lo descrito con bisturí frio y bajo visión endoscópica, excepto en el primer caso, en el cual se realizó con electrobisturí, elemento abandonado posteriormente debido a la presencia de gran cantidad de costras posoperatorias en la fosa.

Se logró la aplicación del test a todos los pacientes incluidos en este estudio, éste fue llevado a cabo por una tecnóloga médico perteneciente al Servicio de ORL.

Durante la evaluación olfativa se obtuvo el siguiente resultado:

- De los 14 pacientes, se objetivaron la mantención de la función olfativa durante el primer mes en 11 pacientes, en 2 restantes hubo una disminución de uno a dos puntos, lo cual llegaron a su estado preoperatorio en los próximos controles.

- 1 paciente $(\mathrm{AA})$ presentó un parámetro de siete en el preoperatorio, el cual bajó a dos en las siguientes evaluaciones. Esta hiposmia se puede explicar, pues en ella se debió realizar varias actividades quirúrgicas rinológicas complementarias como septoplastía y turbinectomía media izquierda (Tabla 2).

Tabla 2.

\begin{tabular}{|llllll|}
\hline Nombre & Edad & Preop. & 1 mes & 3 mes & 6 mes \\
\hline CM & 63 & 5 & 6 & 4 & 5 \\
PF & 54 & 6 & 5 & 5 & 4 \\
JC & 52 & 3 & 6 & 5 & 6 \\
LS & 66 & 5 & 6 & 5 & 7 \\
HM & 58 & 5 & 5 & 5 & 6 \\
AP & 43 & 6 & 7 & 7 & 7 \\
GH & 35 & 6 & 7 & 6 & 6 \\
DM & 54 & 3 & 4 & 4 & 4 \\
ZN & 43 & 7 & 6 & 6 & 6 \\
MH & 43 & 6 & 5 & 5 & 6 \\
TH & 65 & 4 & 3 & 5 & 4 \\
CA & 35 & 5 & 4 & 1 & 4 \\
GV & 57 & 5 & 5 & 5 & 6 \\
AA & 36 & 7 & 2 & 2 & 2 \\
& & & & & \\
\hline
\end{tabular}

\section{DISCUSIÓN}

La elaboración del colgajo nasoseptal dentro de una cirugía transesfenoidal endoscópica para la resección de tumoraciones hipofisiarias, es una técnica relativamente sencilla, con la que se logra el cierre del defecto óseo generado posterior a la extracción del tumor, tal como se grafica en las siguientes imágenes (Figuras 5, 6 y 7).

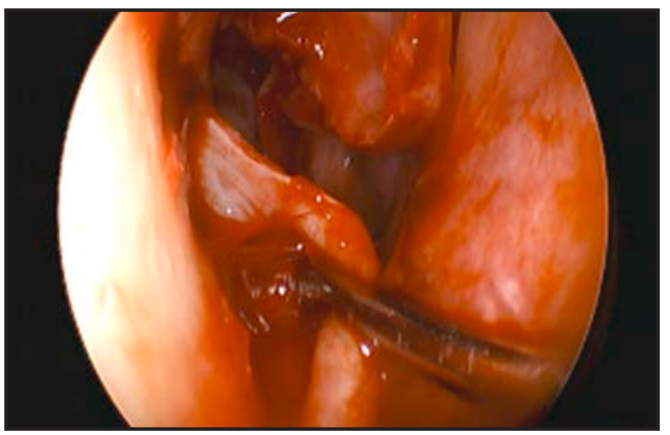

Figura 5. Elevación de colgajo mucopericóndrico.

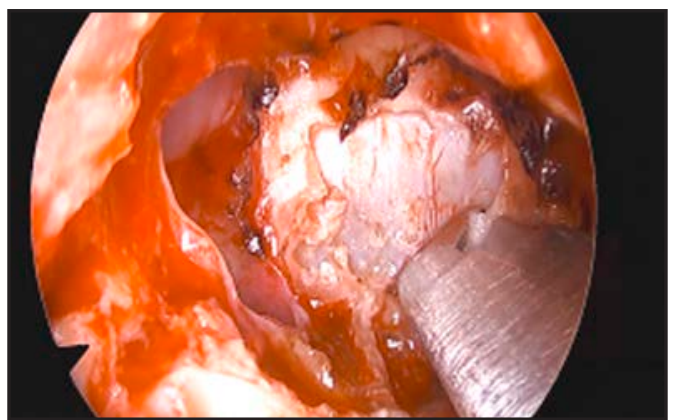

Figura 6. Visualización del lecho hipofisiario.

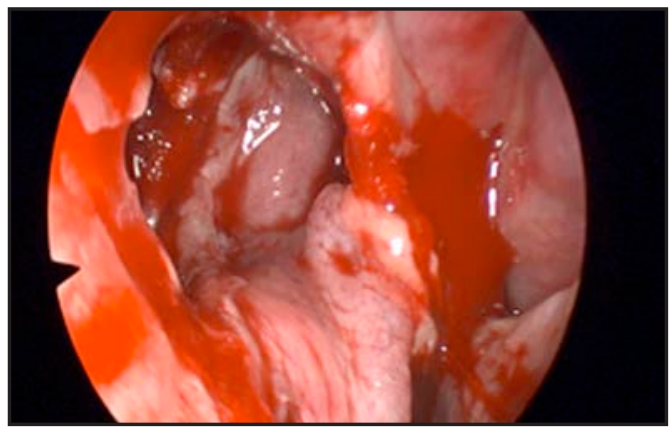

Figura 7. Elaboración de colgajo y cierre del defecto óseo. 
Hemos tomado la decisión de realizar el colgajo a todos los pacientes, estando aun en etapa inicial de nuestro estudio y a su vez en fase aún de aprendizaje, sumado a que tenemos un alto porcentaje de abordaje en recidivas tumorales en los cuales se requieren ciertas medidas especiales $^{16}$, entre otras la forma del cierre de la brecha ósea.

Como forma de prevenir complicaciones rinológicas, hemos diferido levemente de la técnica original ${ }^{9}$ disecando el borde superior de nuestro colgajo a aproximadamente $3 \mathrm{~cm}$. del techo de la fosa y en todos los pacientes hemos mantenido el cornete superior, el cual ha sido sindicado como una de las principales causas de las alteraciones olfatorias ${ }^{17}$. A su vez a diferencia de la técnica descrita por Hadad, quienes realizan el colgajo con electrobisturí, nuestra experiencia fue mejor al utilizar bisturí frio dado que con el monopolar y a pesar de utilizar punta fina, se generaron abundantes costras en la fosa nasal difíciles de manejar en el posoperatorio ${ }^{13}$.

Para la medición del olfato existe varias técnicas, pero en nuestro servicio hemos decidido implementar una metodología sencilla, fácilmente aplicables y que presupuestariamente no sea onerosa ${ }^{15}$. Si bien, la experiencia con este método no es universal, hemos considerado que en este tipo de paciente ha resultado ser útil y podría ser homologable con otras experiencias. La variabilidad individual del resultado del test no ha sido medida pero la apreciación de los autores permite suponer una variabilidad normal positiva o negativa en uno dos puntos.

La hiposmia/anosmia es uno de los efectos secundarios a la técnica de resección vía endoscópica no del todo aclarado, conllevando problemas potencialmente nocivos, como ingestión de alimentos en mal estado, así como incapacidad en detección de fugas de gases o humo de fuego, de igual forma deterioro en su calidad de vida, incluso cuadros de depresión ${ }^{18}$ e incapacidad de degustar alimentos.

En distintos estudios se han evidenciado deterioro olfativo. Mediciones de percepciones subjetivas del olfato como los publicados por Zada $^{19}$. Dusick ${ }^{20}$ muestran deterioros entre 9\% a $10 \%$ de los pacientes sometidos a esta cirugía.
Ikeda ${ }^{21}$ usando mediciones validadas sitúa la pérdida olfativa en $12 \%$.

Kahilogullari ${ }^{22}$ compara 2 grupos, el primero con abordaje microscópico y el segundo endoscópico, sin realizar colgajo en ninguno de ellos, comprueba la presencia de alteraciones olfatorias en el grupo microscópico en forma estadísticamente significativa, dadas fundamentalmente por la presencia de sinequias y el trauma provocado sobre la mucosa nasal.

Rotenberg $^{12}$ en un estudio sobre 17 casos, en los cuales empleó el colgajo nasoseptal, informa deterioro de distinto nivel en todos sus pacientes.

En nuestro país, Vega ${ }^{22}$, no evidenció deterioro de este sentido tras la realización de la técnica endoscópica, si bien no se menciona la realización de colgajos.

En nuestro estudio, si bien es limitado en número de pacientes y aun inicial, no hemos encontrado deterioro significativo en la calidad del sentido del olfato en éstos. Así como tampoco hemos observado otras complicaciones como necrosis del colgajo, infecciosas significativas $\mathrm{y} / 0$ vasculares.

Sabemos que el número de pacientes incluidos en este estudio no alcanza a las series que han reportado alteración olfativa posterior a la resección hipofisiaria transesfenoidal endoscópica, lo que pudiese ser un sesgo. Pero debemos considerar que esta patología tiene una frecuencia relativa, sin embargo gracias a los grupos interdisciplinarios como el nuestro la casuística ha ido en aumento, al ser considerados como centro de derivación.

Es importante remarcar que la resección endoscópica presenta menos efectos adversos que las técnicas tradicionales de resección hipofisiaria y es nuestro objetivo demostrar que existe menos impacto en la olfación con este método. Por otra parte las técnicas de medición de olfación descritas y sobre todo la utilizada en este estudio, son aceptablemente objetivas, lo que pudiese explicar los buenos resultados demostrados casi en la totalidad de los pacientes, siendo interesante estudiar y homologar a alguna técnica más universal de medición de la olfación. 


\section{CONCLUSIÓN}

Nuestro estudio, siendo aún un trabajo inicial como indicamos, adolece de poseer un número limitado de pacientes, pero estimamos que podría ya esbozar una

\section{BIBLIOGRAFÍA}

1. Sethi DS, PlLAY PK. Endoscopic management of lesions of the sella turcica. J Laryngol Otol1995; 109: 956-62.

2. Jho HD, Carrau RL, Ko Y. Endoscopic pituitary surgery. En: Wilkins RH, Rengachary SS, editores. Neurosurgical operative atlas. Park Ridge: American Association of Neurological Surgery; 1996. p. 1-12.

3. Carrau RL, Jho hD, Ko Y. Transnasaltranssphenoidal endoscopic surgery of the pituitary gland. Laryngoscope 1996; 106: 914-8.

4. LIU JK, KAUSHIK D, WEISS MH, ET AL. The history and evolution of transsphenoidal surgery. $J$ Neurosurg 2011; 95: 1083-96.

5. Kennedy DW, Cohn ES, PaPe ID, et al. Transsphenoidal approach to the sella: the Johns Hopkins experience. Laryngoscope 1984; 94: 1066-74.

6. Sariego H, Bogado M. Evaluación nasal en el abordaje quirúrgico de la patología selar. Rev Otorrinolaringol Cir Cabeza Cuello 2008; 68: 164-70.

7. Olavarría C, Stott C, Lemp M, Bustamante C, Schmidt N, Emerich M. Comparación de dos técnicas quirúrgicas para abordaje de la región selar: Transeptal transesfenoidal versus transnasal directa. Rev Otorrinolaringol Cir Cabeza Cuello 2004; 64: 91-8.

8. Arias R, Gómez G, Rojas M. Descripción de variantes del acceso transeptoesfenoidal para el abordaje de la patología selar. Rev Otorrinolaringol Cir Cabeza Cuello 2014; 74: 75-80.

9. Hadad G, Bassagasteguy L, Carrau Rl, Mataza JC, Kassam A, Snyderman CH, et al. A novel tendencia de mantención la función olfativa en este tipo de cirugía.

A su vez tenemos los desafíos de optimizar nuestra experiencia, como agregar un grupo control y un test homologables en forma más universal. Todo lo anterior representa nuevos retos para nuestro equipo.

reconstructive technique following endoscopic expanded endonasal approaches: Vascular pedicle nasoseptal flap. Laryngoscope 2006; 116: 1882-6.

10. Rivera-Serrano CM, Snyderman CH, Gardner P, ET AL. Nasoseptal "rescue" flap: A novel modification of the nasoseptal flap technique for pituitary surgery. Laryngoscope $2011 ; 121$ : 990-3.

11. Hart CK, Theodosopoulos PV, and Zimmer LA. Olfactory changes after endoscopic pituitary tumor resection. Otolaryngol Head Neck Surg 2010; 142: 95-97.

12. Rotenberg BW, Rotenberg, Saunders Saunders, Duggal N. Olfactory Outcomes After Endoscopic Transsphenoidal Pituitary Surgery. Laryngoscope 2011; 121: 1611-3.

13. Hong S, Nam H, Park J, et al. Olfactory outcomes after endoscopic pituitary surgery with nasoseptal "rescue" flaps: Electrocautery versus cold knife. Am J Rhinol Allergy 2014; 28: 517-9.

14. Hummel T, Nordin S. Olfactory disorders and their consequences for quality of life a review. Acta Otolaryngol 2005; 125: 116-21.

15. C Der M, F Larach F, N Hananías P, M Cohen V, MP Salin, A Mesin. Olfatómetro práctico: Propuesta de una nueva herramienta clínica. Rev Otorrinolaringol Cir Cab-Cuello 2002; 62: 129-36.

16. P Cappabianca, A Alfieri, A Colao, LM Cavallo, M Fusco, C Peca, G Lombardi. Endoscopic Endonasal Transsphenoidal Surgery in Recurrent and Residual Pituitary Adenomas: Technical Note. E. de Divitiis. Minim Invas Neurosurg 2000; 43: 38-43.

17. Orhan M, Govsa F, Saylam C. A surgical view of the superior nasal turbinate. Eur Arch Otorhinolaryngol 2010; 267: 909-16. 
18. Smeets MA, Veldhuizen MG, Galle S, et al. Sense of smell disorder and health-related quality of life. Rehabil Psychol 2009; 54: 404-12.

19. Zada G, Kelly D, Cohan P, et al. Endonasal transsphenoidal approach for pituitary adenomas and other sellar lesions: an assessment of efficacy, safety, and patient impressions. $J$ Neurosurg 2003; 98: 350-8.

20. Dusick J, Espósito F, Mattozo C, et al. Endonasal transsphenoidal surgery: the patient's perspective-survey results from 259 patients. Surg Neurol 2006; 65: 332-42.

21. Ikeda K, Watanabe K, Suzuki H, et al. Nasal airway resistance and olfactory acuity following transsphenoidal pituitary surgery. Am J Rhinol 1999; 13: 45-8.

22. Kahilogullari G, Beton $S$, Al-Beyati E, Kantarcioglu 0, Bozkurt M, Kantarcioglu E, Comert A, Untu M A, Meco C. Olfactory functions after transsphenoidal pituitary surgery: Endoscopic versus microscopic approach. Laryngoscope 2013; 123: 2112-9.

22. Vega N, Walker K, Boettiger J. Evaluación del olfato en pacientes con patología tumoral en la región selar, intervenidos mediante abordaje endoscópico transesfenoidal transnasal. Rev Otorrinolaringol Cir Cabeza Cuello 2013; 73: $133-9$. 